TRANSACTIONS OF THE

AMERICAN MATHEMATICAL SOCIETY

Volume 349, Number 7, July 1997, Pages 2969-2980

S 0002-9947(97)01696-6

\title{
SUBGROUPS OF FINITE SOLUBLE GROUPS INDUCING THE SAME PERMUTATION CHARACTER
}

\author{
NORBERTO GAVIOLI
}

\begin{abstract}
In this paper there are found necessary and sufficient conditions that a pair of solvable finite groups, say $G$ and $K$, must satisfy for the existence of a solvable finite group $L$ containing two isomorphic copies of $G$ and $H$ inducing the same permutation character. Also a construction of $L$ is given as an iterated wreath product, with respect to their actions on their natural modules, of finite one-dimensional affine groups.
\end{abstract}

\section{INTRODUCTION}

We shall say that two subgroups $G$ and $H$ of a finite group $L$ are linked (in $L$ ) if the characters of $L$ induced by the principal characters of $G$ and $H$ are equal. Equivalently, $G$ and $H$ are linked in $L$ if $|C \cap G|=|C \cap H|$ for each conjugacy class $C$ of elements in $K$. It is obvious that conjugate subgroups of $K$ are linked, but linked subgroups need not even to be isomorphic (see [1], [3], [4] and [8]). This fact has been used to give examples of pairs of objects which have some similar properties but are not isomorphic. In [8] pairs of nonconjugate linked subgroups of a finite group are used to construct examples of arithmetically equivalent number fields which are not isomorphic. In [9] pairs $(H, K)$ of nonisomorphic linked subgroups provide examples of pairs of isospectral riemannian manifolds which are not isometric; indeed it turns out that $H$ and $K$ are the fundamental groups of these manifolds. A natural question is: for which pairs of finite groups $G$ and $H$ there exists a group $L$ with linked subgroups isomorphic to $G$ and $H$ ?

It is easy to see that, if $G$ and $H$ are linked subgroups of $L$, then, for each $n$, the number of elements of order $n$ in $G$ is the same as in $K$. Conversely assume that $G$ and $H$ are finite groups satisfying this condition. Then $G$ and $H$ have the same number of elements, so we can embed $G$ and $H$ as regular subgroups of one suitable symmetric group. The images of these embeddings are linked subgroups in that symmetric group (this result appears in [8]).

Assume now that $G$ and $H$ are linked subgroups of a $p$-group $L$. Choose a chief series for $L$ and a generator for each of the chief factors of the series. This is called a Sylow sequence for $L$. Intersecting this series with $G$ and $H$ and eliminating repetitions, one finds Sylow sequences for $G$ and $H$. If $g$ is an element of $G$, note which chief factor it 'lives' in and which power of the generators it projects to. Repeat the same for $g^{p}, g^{p^{2}}$, and so on. The result is the weight-leader sequence

Received by the editors October 7, 1994 and, in revised form, January 4, 1996.

1991 Mathematics Subject Classification. Primary 20C99, 20 D10.

Key words and phrases. Permutation character of finite groups, finite solvable groups.

Work partially supported by M.U.R.S.T. 
for $g$. Since $G$ and $H$ are linked in $L$ and their Sylow sequences are obtained from that of $L$, it follows that, for each prescribed weight-leader sequence $w l$, the groups $G$ and $H$ have the same number of elements having weight-leader sequence equal to $w l$. Conversely if $G$ and $H$ satisfy this condition with respect to suitable Sylow sequences, then there exists a $p$-group $L$ with linked subgroups isomorphic to $G$ and $H$. The group $L$ can be chosen to be a Sylow subgroup of the symmetric group on $|H|$ letters (see [1]).

This paper provides a similar condition for answering the question: given two finite solvable groups $G$ and $H$, is there a finite solvable group with linked subgroups isomorphic to $G$ and $H$ ? Instead of considering chief series, we define a 'weight sequence' of an element of $G$ with respect to a series of normal subgroups with abelian factors of prime exponent. To each $g$, note which factor it lives in; if $p$ is the exponent of that quotient do the same with $g^{p}$, and so on. The result of this operation is the weight sequence of $g$. As in the case of $p$-groups, if $G$ and $H$ are linked subgroups of a finite solvable group, choose a chief series for $L$. Forming intersections with $G$ and $H$, obtain series of normal subgroups of $G$ and $K$ and define the weight sequences in terms of these. It is easy to see that for each prescribed weight sequence $w$, the groups $G$ and $H$ have the same number of elements having weight sequence equal to $w$. Conversely assume that $G$ and $H$ are two finite solvable groups satisfying the above condition. One can easily see that $|G|=|H|$, that the two series have the same length and that the corresponding factors have the same orders. In particular $G$ and $H$ can be embedded in the same symmetric group as regular subgroups. The orbits of the subgroups in the series of $G$ form a tree of imprimitivity blocks for the action of $G$. The normalizer of this block tree is the iterated wreath product of the symmetric groups acting on the factors of the series of $G$. Indeed $G$ is contained in a smaller group $W$ which is the iterated wreath product, with respect to the regular action, of the elementary abelian factors of the series of $G$. Since two such groups are conjugate in the symmetric group on $|G|$ letters, we can assume that both $G$ and $H$ are subgroups of the same $W$. However in Section 5 we give an example in which $G$ and $H$ need not be linked in $W$ for every choice of series for $G$ and $H$ as above. So we choose a larger iterated wreath product. Regard the elementary abelian group of order $p^{k}$ as the translation subgroup of the one-dimensional affine group $A\left(p^{k}\right)$ over the Galois field $\mathbb{F}\left(p^{k}\right)$. Let $L$ be the iterated wreath product of these affine groups acting on the factors of the series of $G$ (and of $H$ ), then $G$ and $H$ are embedded in $L$ as linked subgroups.

This result is part of my Ph.D. dissertation that has been written in Italian at the University of Trento under the supervision of Professor A. Caranti. I want to thank him for his assistance. I want also to thank the referee for his helpful suggestions for the revision of this paper and also for improving and shortening the arguments contained in Section 5 .

\section{Notation}

Let $G$ be a soluble group. An invariant series of $G$ is a series of normal subgroups of $G$. Let

$$
\Sigma: 1=G_{0} \leq G_{1} \leq \cdots \leq G_{n}=G
$$

be an invariant series of $G$ with abelian factors of prime exponent. Let $V_{i}$ be the quotient $G_{i} / G_{i-1}$ and $p_{i}$ the exponent of $V_{i}$. We put also $p_{0}=1$. For every $i=1, \ldots, n$ we denote by $\pi_{i}: G_{i} \rightarrow V_{i}$ the projection on the quotient and we 
choose a transversal $\mathrm{T}_{i}: V_{i} \rightarrow G_{i}$ for $G_{i-1}$ in $G_{i}$ such that $\mathrm{T}_{i}(0)=1$; we shall call it a restricted transversal.

The direct product $D_{1, n}=V_{1} \times \cdots \times V_{n}$ will be called the tangent space of $G$ with respect to $\Sigma$. We will use the additive notation for the abelian groups $V_{i}$ and $D_{1, n}$.

We define also a restricted "exponential" function $\mathrm{T}: D_{1, n} \rightarrow G$ by letting

$$
\mathrm{T}\left(v_{1}, v_{2}, \ldots, v_{n}\right)=\mathrm{T}_{1}\left(v_{1}\right) \cdot \mathrm{T}_{2}\left(v_{2}\right) \cdots \mathrm{T}_{n}\left(v_{n}\right) .
$$

It is easy to verify that the function $\mathrm{T}$ is bijective, with an inverse $\log : G \rightarrow D_{1, n}$.

If $g \in G$ we define the weight of $g$ with respect to the series $\left\{G_{i}\right\}_{i=0}^{n}$ to be the nonnegative integer $\operatorname{wt}(g)=\min \left\{i \mid g \in G_{i}\right\}$.

\section{WREATH PRODUCTS OF FINITE ELEMENTARY ABELIAN GROUPS}

Let $A$ and $B$ be two groups and let $Y$ be a $B$-set; then $B$ acts on $A^{Y}$ as an automorphism group by means of the coinduced action: $f^{b}(y)=f\left(y^{b^{-1}}\right)$.

Definition 1. The wreath product $A_{\ell_{Y}} B$ is the semidirect product $B\left[A^{Y}\right]$ with respect to the coinduced action. When $Y$ is $B$ itself seen as a $B$-space via the regular action, then we shall write, for short, $A \imath_{2} B$ in place of $A \imath_{B} B$.

The elements of $A \imath_{Y} B$ are represented by pairs $(b, f)$, where $b \in B$ and $f \in A^{Y}$. The group $A^{Y}$ may be identified with the subgroup $\left\{(1, f): f \in A^{Y}\right\}$ of $A \ell_{Y} B$, which is called the base subgroup of $A \lambda_{Y} B$ and it is the kernel of the canonical projection $B\left[A^{Y}\right] \rightarrow B$. If $A$ and $B$ are groups acting respectively on the sets $X$ and $Y$, then there exists a canonical action of $A$ ใ $_{Y} B$ over $X \times Y$ defined by: $(x, y)^{(b, f)}=\left(x^{f\left(y^{b}\right)}, y^{b}\right)$. It is easy to see that this action is faithful if and only if $X$ and $Y$ are faithful respectively as an $A$-set and a $B$-set.

Lemma 2. Let $A$ and $B$ be two transitive permutation groups acting respectively on $X$ and $Y$, where $|X|,|Y|>1$. Then $G=A l_{Y} B$ is imprimitive on the set $X \times Y$. Moreover the fibers of the projection $\pi_{Y}: X \times Y \rightarrow Y$ are imprimitivity blocks and the kernel of the action of $G$ on the set of the blocks is the base subgroup $A^{Y}$ of $G$.

Proof. The set $Y$ is a $G$-set by letting $y^{(b, f)}=y^{b}$. The projection $\pi_{Y}(x, y)=y$ is then a $G$-function and its fibers are trivially imprimitivity blocks for the $G$-action. The stabilizer of the fiber $\pi_{Y}^{-1}(y)$ is the subgroup $G_{\pi_{Y}^{-1}(y)}=\left\{(b, f) \in G: y^{b}=y\right\}$. Hence the kernel of the action of $G$ on the fibers is $N=\bigcap\left\{G_{\pi_{Y}^{-1}(y)}: y \in Y\right\}$. Since $B$ acts faithfully on $Y$, then $N$ is the base subgroup of $G$.

The next lemma is well known and the proof will be omitted.

Lemma 3. Let $A, B$ and $C$ be permutation groups acting respectively on the sets $X, Y$ and $Z$. Then the set-isomorphism $(X \times Y) \times Z \cong X \times(Y \times Z)$ yields an equivalence of permutation groups:

$$
\left(A \iota_{Y} B\right) \imath_{Z} C \cong A \imath_{Y \times Z}\left(B \imath_{Z} C\right) .
$$

Let $\left\{V_{i}\right\}_{i=1}^{\infty}$ be a sequence of finite abelian groups of prime exponents $p_{i}=$ $\exp \left(V_{i}\right)$. Then we shall use the following notation

- $\mathcal{G}_{h, k}= \begin{cases}{ }_{i=h}^{k} V_{i}=\left(\cdots\left(V_{h} \prec V_{h+1}\right) \prec \cdots\right) \prec V_{k} & \text { if } h \leq k, \\ 1 & \text { if } h>k\end{cases}$ 
- $D_{h, k}= \begin{cases}\prod_{i=h}^{k} V_{i}=V_{h} \times \cdots \times V_{k} & \text { if } h \leq k \\ 0 & \text { if } h>k\end{cases}$

- $\mathcal{M}_{h, j, k}=\left(\mathcal{G}_{h, j}\right)^{D_{j+1, k}}$ for $1 \leq h \leq j \leq k$.

For short, we shall denote the group $\mathcal{G}_{1, n}$ by $\mathcal{G}_{n}$. The group $\mathcal{G}_{h, k}$ is clearly a permutation group on $D_{h, k}$.

Lemma 4. If $h<k<j$, then the canonical set-isomorphism $\left(V_{h} \times \cdots \times V_{j-1}\right) \times$ $V_{j} \cong\left(V_{h} \times \cdots \times V_{k}\right) \times\left(V_{k+1} \times \cdots \times V_{j}\right)$ yields an equivalence of permutation groups:

$$
\mathcal{G}_{h, j} \cong \mathcal{G}_{k+1, j}\left[\mathcal{M}_{h, k, j}\right]=\mathcal{G}_{h, k} \imath_{D_{k+1, j}} \mathcal{G}_{k+1, j} .
$$

Proof. It is an inductive consequence of Lemma 3.

Proposition 5. If $k>h$, then $\mathcal{G}_{h, k}$ is imprimitive on $D_{h, k}$. Moreover the following statements hold:

(i) If $h \leq j<k$ and $\left(v_{j+1}, \ldots, v_{k}\right) \in D_{j+1, k}$, then the set $B\left(v_{j+1}, \ldots, v_{k}\right)=$ $D_{h, j} \times\left\{\left(v_{j+1}, \ldots, v_{k}\right)\right\} \subseteq D_{h, k}$ is an imprimitivity block for $\mathcal{G}_{h, k}$,

(ii) if $h \leq j<k$, then $\mathcal{G}_{h, k}$ acts transitively on the set $F_{j, k} \cong D_{j+1, k}$ of blocks of the form $B\left(v_{j+1}, \ldots, v_{k}\right)$ and this action has kernel $\mathcal{M}_{h, j, k}$,

(iii) $\mathcal{G}_{h, k}$ is equivalent, as a permutation group, to $\mathcal{G}_{j+1, k}\left[\mathcal{M}_{h, j, k}\right]$,

(iv) $\mathcal{G}_{h, k} \cong \mathcal{M}_{k, k, k}\left[\mathcal{M}_{k-1, k-1, k}\left[\mathcal{M}_{k-2, k-2, k}\left[\cdots\left[\mathcal{M}_{h, h, k}\right] \cdots\right]\right]\right]$.

Proof. Points (i), (ii) and (iii) follow from Lemmas 4 and 2 applied to the groups $G=\mathcal{G}_{h, k} \cong \mathcal{G}_{h, j} ?_{D_{j+1, k}} \mathcal{G}_{j+1, k}$. In particular the group $\mathcal{G}_{h, k}$ acts on the blocks of $F_{h, k}$ with kernel $\mathcal{M}_{h, h, k}$ and is equivalent to $\mathcal{G}_{h+1, k}\left[\mathcal{M}_{h, h, k}\right]$. Hence, arguing by induction on $k-h$ we obtain the result of statement (iv).

When $h=1$ and $k=n$ we put, for short, $\mathcal{B}_{j}=\mathcal{M}_{1, j, n}$ and $\mathcal{V}_{j}=\mathcal{M}_{j, j, n} \leq \mathcal{G}_{n}=$ $\mathcal{G}_{1, n}$. Note that $\mathcal{B}_{j} \leq \mathcal{B}_{j+1}$ and since $\mathcal{G}_{1, n}$ splits over $\mathcal{B}_{j}$ so does $\mathcal{B}_{j+1}$. The factor $\mathcal{B}_{j+1} / \mathcal{B}_{j}$ corresponds to $\mathcal{V}_{j+1}$ in the canonical isomorphism $\mathcal{G}_{j+1, n} \cong \mathcal{G}_{1, n} / \mathcal{B}_{j}$, i.e. to the kernel of $\mathcal{G}_{j+1, n}$ acting on $F_{j+2, n}$. We then have $\mathcal{B}_{j+1}=\mathcal{V}_{j+1}\left[\mathcal{B}_{j}\right]$. As a consequence we find the following equalities for $k=1, \ldots, n$ :

$$
\mathcal{B}_{k}=\mathcal{V}_{k} \cdot \mathcal{V}_{k-1} \cdots \mathcal{V}_{1}=\mathcal{V}_{k}\left[\mathcal{B}_{k-1}\right]
$$

Let $g, g^{\prime} \in \mathcal{G}_{n}$, by the previous equalities we can write $g=m_{n} \cdot m_{n-1} \cdots m_{1}$ and $g^{\prime}=m_{n}^{\prime} \cdot m_{n-1}^{\prime} \cdots m_{1}^{\prime}$, with $m_{i}, m_{i}^{\prime} \in \mathcal{V}_{i}$. So $m_{i}$ is identified with a function $m_{i}: D_{i+1, n} \rightarrow V_{i}$. The action of $\mathcal{G}_{n}$ over $D_{1, n}$ is then given by $\left(v_{1}, \ldots, v_{n}\right)^{g^{-1}}=$ $\left(v_{1}-m_{1}\left(v_{2}, \ldots, v_{n}\right), \ldots, v_{n-1}-m_{n-1}\left(v_{n}\right), v_{n}-m_{n}\right)$. Moreover for $i<j$ we have the formula:

$$
m_{i}^{m_{j}^{\prime}}\left(v_{i+1}, \ldots, v_{n}\right)=m_{i}\left(v_{i+1}, \ldots, v_{j-1}, v_{j}-m_{j}^{\prime}\left(v_{j+1}, \ldots, v_{n}\right), v_{j+1}, \ldots, v_{n}\right)
$$

which can be used to describe the group operation when the elements of $\mathcal{G}_{n}$ are represented in the above form.

The invariant series of $\mathcal{G}_{n}$

$$
\Omega: 1=\mathcal{B}_{0} \leq \mathcal{B}_{1} \leq \cdots \leq \mathcal{B}_{n}=\mathcal{G}_{n}
$$

will be called the canonical series of $\mathcal{G}_{n}$. Its factor groups are the elementary abelian groups $\mathcal{V}_{k}$. The weight of an element of $\mathcal{G}_{n}$ will always be computed with respect to this series. For $h \leq j \leq k$, denote the permutation representation given by the action of $\mathcal{G}_{h, k}$ over $F_{j, k} \cong D_{j+1, k}$, described in point (ii) of the previous proposition, by $\rho_{j, k}: \mathcal{G}_{h, k} \rightarrow \mathcal{G}_{j+1, k}$ and put also $\rho_{h-1, k}=$ id. 
Definition 6. We say that an element $g \in \mathcal{G}_{h, k}$ is diagonal if, for all integers $j$ such that $h-1 \leq j<k$, the group $\left\langle\rho_{j, k}(g)\right\rangle$ is either a trivial or a semiregular subgroup of $\mathcal{G}_{j+1, k}$.

Proposition 7. Let $0<s<n$ be two positive integers and $g \in \mathcal{B}_{s} \leq \mathcal{G}_{n}$ with $g \neq 1$. Considering the decomposition $\mathcal{G}_{n}=\mathcal{G}_{s} 2_{D_{s+1, n}} \mathcal{G}_{s+1, n}$ and writing $g=(1, a)$, with $a: D_{s+1, n} \rightarrow \mathcal{G}_{1, s}$, the following are equivalent:

(i) $g$ is a diagonal element of $\mathcal{G}_{n}$,

(ii) for all $d \in D_{s+1, n}$ the element $a(d) \in \mathcal{G}_{s}$ is diagonal and $\operatorname{wt}\left(g^{k}\right)=\operatorname{wt}\left(a(d)^{k}\right)$ for all $k \in \mathbb{Z}$.

Proof. (i) $\Rightarrow$ (ii). Let $d=\left(v_{s+1}, \ldots, v_{n}\right) \in D_{s+1, n}$ and suppose that $\operatorname{wt}\left(a(d)^{k}\right)=$ $t \leq s$. Then, for all choices of $v_{i} \in V_{i}$, where $i=t+1, \ldots, s$, the set $B\left(v_{t+1}, \ldots, v_{s}\right)$ $=D_{1, t} \times\left(v_{t+1}, \ldots, v_{s}\right)$ is a permutation block fixed by $\rho_{t, s}\left(a(d)^{k}\right)$. Hence $\rho_{t, n}\left(g^{k}\right)$ fixes the block $B\left(v_{t+1}, \ldots, v_{s}, d\right)=D_{1, t} \times\left(v_{t+1}, \ldots, v_{n}\right) \in F_{t, n}$. Since $g$ is diagonal, we have $\rho_{t, n}\left(g^{k}\right)=1$, which is equivalent to $\operatorname{wt}\left(g^{k}\right) \leq t=\operatorname{wt}\left(a_{d}^{k}\right)$. So $\operatorname{wt}\left(g^{k}\right) \leq$ $\min \left\{\operatorname{wt}\left(a(d)^{k}\right): d \in D_{s+1, n}\right\}$. On the other hand, if $B\left(v_{t+1}, \ldots, v_{s}\right)$ is a block which is not fixed by $\rho_{t, s}\left(a(d)^{k}\right)$, then $B\left(v_{t+1}, \ldots, v_{s}, d\right)$ is not fixed by $g$ and so $\operatorname{wt}\left(g^{k}\right) \geq \max \left\{\operatorname{wt}\left(a(d)^{k}\right): d \in D_{s+1, n}\right\}$. Hence $\operatorname{wt}\left(g^{k}\right)=\operatorname{wt}\left(a(d)^{k}\right)$ for all $k \in \mathbb{Z}$ and for all $d \in D_{s+1, n}$. To see that $a(d)$ is diagonal let $k \in \mathbb{Z}$. Assuming that $t \leq s$ is a positive integer for which there exists a block $B\left(v_{t+1}, \ldots, v_{s}\right)$ fixed by $\rho_{t, s}\left(a(d)^{k}\right)$, we want to show that $\rho_{t, s}\left(a(d)^{k}\right)=1$. In fact $B\left(v_{t+1}, \ldots, v_{n}\right)=$ $B\left(v_{t+1}, \ldots, v_{s}\right) \times d=B\left(v_{t+1}, \ldots, v_{s}\right)^{a(d)^{k}} \times d=B\left(v_{t+1}, \ldots, v_{n}\right)^{g^{k}}$ implies that $B\left(v_{t+1}, \ldots, v_{n}\right)$ is a block fixed by $\rho_{t, s}\left(g^{k}\right)$ and, since $g$ is diagonal, $\rho_{t, s}\left(g^{k}\right)=1$. Thus $\operatorname{wt}\left(a(d)^{k}\right)=\operatorname{wt}\left(g^{k}\right) \leq t$, which is equivalent to $\rho_{t, s}\left(a(d)^{k}\right)=1$.

(ii) $\Rightarrow$ (i). Let $t \leq s$ and $k \in \mathbb{Z}$. Assume that $B\left(v_{t+1}, \ldots, v_{n}\right)$ is a block fixed $g^{k}$, we have to show that $\rho_{t, n}\left(g^{k}\right)=1$. Since $\rho_{s, n}\left(g^{k}\right)=1$, without loss of generality we can assume that $t \leq s$. Put $d=\left(v_{s+1}, \ldots, v_{n}\right)$, then $B\left(v_{t+1} \ldots, v_{s}\right) \times d=$ $B\left(v_{t+1}, \ldots, v_{n}\right)=B\left(v_{t+1}, \ldots, v_{n}\right)^{g^{k}}=B\left(v_{t+1} \ldots, v_{s}\right)^{a_{d}^{k}} \times d$. So the element $\rho_{t, s}\left(a(d)^{k}\right)$ fixes the block $B\left(v_{t+1} \ldots, v_{s}\right)$. Since $a(d)$ is diagonal, we must have $\rho_{t, s}\left(a(d)^{k}\right)=1$. We deduce $\operatorname{wt}\left(g^{k}\right)=\operatorname{wt}\left(a(d)^{k}\right) \leq t$, which is equivalent to $\rho_{t, n}\left(g^{k}\right)=1$.

Let $V$ be a finite abelian group of prime exponent $p$. Let $\phi: A \imath V \rightarrow V$ be the canonical projection.

Proposition 8. With the above notation if $g, g^{\prime} \in A<V$ are such that $\phi(g) \neq 0$, then the following are equivalent:

(i) $g$ and $g^{\prime}$ are conjugate in $A$ ? $V$;

(ii) $g^{p}$ and $g^{\prime p}$ are conjugate in $A \prec V$ and $\phi(g)=\phi\left(g^{\prime}\right)$.

Proof. The only nontrivial implication is (ii) $\Rightarrow$ (i).

Assume (ii). Then there exists an element $v \in V$ such that $g^{p}$ and $\left(g^{\prime p}\right)^{v}$ are conjugate elements in the group $A^{V}$. Let $C_{p}=\langle\phi(g)\rangle$ and write $W=C_{p} \times W$ for a suitable subgroup $W$ of $V$. Note that $g, g^{\prime v} \in C_{p}\left[A^{V}\right]=A^{W}\left\{C_{p}\right.$. From [1], Lemma 2, it follows that $g$ and $g^{\prime v}$ are conjugate elements in $C_{p}\left[A^{V}\right]$, which implies that $g$ and $g^{\prime}$ are conjugate elements in $A$ ? $V$.

Denote by $G$ a finite soluble group with a given invariant series $\left\{G_{i}\right\}_{i=0}^{n}$ such that $V_{i}=G_{i} / G_{i-1}$ is abelian of exponent $p_{i}$. The group $\mathcal{G}_{n}$ acts on $D_{1, n}=\prod_{i=1}^{n} V_{i}$, hence the set $S(G)=G^{D_{1, n}}$ is a $\mathcal{G}_{n}$-set via the coinduced action. There exists 
a bijection between the set $M(G)$, whose elements are $n$-tuples $\left(\mathrm{T}_{1}, \ldots, \mathrm{T}_{n}\right)$ of restricted transversals $\mathrm{T}_{i}: V_{i} \rightarrow G_{i}$, and the subset $E(G)$ of $S(G)$, whose elements are the restricted exponential functions. In fact the function $\psi: M(G) \rightarrow E(G)$ defined by $\left(\psi\left(\mathrm{T}_{1}, \ldots, \mathrm{T}_{n}\right)\right)\left(v_{1}, \ldots, v_{n}\right)=\mathrm{T}_{1}\left(v_{1}\right) \cdot \mathrm{T}_{2}\left(v_{2}\right) \cdots \mathrm{T}_{n}\left(v_{n}\right)=\mathrm{T}\left(v_{1}, \ldots, v_{n}\right)$ has inverse defined by

$$
\left(\psi^{-1}(\mathrm{~T})\right)_{i}\left(v_{i}\right)=\mathrm{T}\left(0, \ldots, 0, v_{i}, 0, \ldots, 0\right) .
$$

We define some new subgroups of $\mathcal{G}_{n}$. For $i \leq n$ put

$$
\mathcal{C}_{i}=\left\{m_{i} \in \mathcal{V}_{i} \mid m_{i}(0, \ldots, 0)=0\right\} .
$$

Then $\mathcal{C}_{i} \leq \mathcal{V}_{i} \leq \mathcal{G}_{n}$. From formula (2) follows $\left[\mathcal{C}_{i}, \mathcal{C}_{j}\right] \leq \mathcal{C}_{i}$ for $i \leq j$. We can then set $\mathcal{E}_{n}=\mathcal{C}_{n-1} \mathcal{C}_{n-2} \cdots \mathcal{C}_{1} \leq \mathcal{G}_{n}$.

Lemma 9. Let $\mathrm{T}$ and $\mathrm{T}^{\prime}$ be two restricted exponential functions, then there exist elements $m_{i} \in \mathcal{C}_{i}$, for $i=1, \ldots, n-1$, such that, for all $\left(v_{1}, \ldots, v_{n}\right) \in D_{1, n}$, there holds

$$
\mathrm{T}^{\prime}\left(v_{1}, \ldots, v_{n}\right)=\mathrm{T}\left(v_{1}-m_{1}\left(v_{2}, \ldots, v_{n}\right), \ldots, v_{n-1}-m_{n-1}\left(v_{n}\right), v_{n}\right) .
$$

Proof. We argue by induction on the number $n$ of factors of the series $\left\{G_{i}\right\}_{i=0}^{n}$. If $n=1$ the claim is easily seen to be true. If $n>1$, let $\mathrm{T}$ and $\mathrm{T}^{\prime}$ be elements of $E(G)$. Consider the function $f: E(G) \rightarrow S\left(G / G_{1}\right)$ defined by:

$$
f(\mathrm{~T})\left(v_{2}, \ldots, v_{n}\right)=\mathrm{T}\left(0, v_{2}, \ldots, v_{n}\right) G_{1} .
$$

Clearly $f(E(G))=E\left(G / G_{1}\right)$. By induction hypotheses there exist $m_{i} \in \mathcal{C}_{i}$, for $i=2, \ldots, n-1$, such that

$$
\begin{aligned}
& \mathrm{T}\left(0, v_{2}-m_{2}\left(v_{3}, \ldots, v_{n}\right), \ldots, v_{n-1}-m_{n-1}\left(v_{n}\right), v_{n}\right) \\
& \quad=c\left(v_{2}, \ldots, v_{n}\right) \mathrm{T}_{2}^{\prime}\left(v_{2}\right), \ldots, \mathrm{T}_{n}^{\prime}\left(v_{n}\right)
\end{aligned}
$$

for some function $c: V_{2} \times \cdots \times V_{n} \rightarrow G_{1}$. The transversal $\mathrm{T}_{1}: V_{1} \rightarrow G_{1}$ is an isomorphism of abelian groups and it is the inverse function of the isomorphism $\pi_{1}: G_{1} \stackrel{\sim}{\rightarrow} V_{1}$. Since the sameIf, holds for $\mathrm{T}_{1}^{\prime}$, we have $\mathrm{T}_{1}=\mathrm{T}_{1}^{\prime}$. Let then $m_{1}=\pi_{1} \circ c$, then $m_{1} \in \mathcal{V}_{1}$. If, for $i=2, \ldots, n$, we let $v_{i}=0$ in equation (5), then we find $c(0, \ldots, 0)=1$, so that $m_{1}$ is an element of $\mathcal{C}_{1}$. We then have

$$
\begin{aligned}
\mathrm{T}\left(v_{1}-\right. & \left.m_{1}\left(v_{2}, \ldots, v_{n}\right), v_{2}-m_{2}\left(v_{3}, \ldots, v_{n}\right), \ldots, v_{n-1}-m\left(v_{n}\right), v_{n}\right) \\
& =\mathrm{T}_{1}\left(v_{1}-m_{1}\left(v_{2}, \ldots, v_{n}\right)\right) c\left(v_{2}, \ldots, v_{n}\right) \mathrm{T}_{2}^{\prime}\left(v_{2}\right) \cdots \mathrm{T}_{n}^{\prime}\left(v_{n}\right) \\
& =\mathrm{T}_{1}\left(v_{1}\right) c\left(v_{2}, \ldots, v_{n}\right)^{-1} c\left(v_{2}, \ldots, v_{n}\right) \mathrm{T}_{2}^{\prime}\left(v_{2}\right) \cdots \mathrm{T}_{n}^{\prime}\left(v_{n}\right) \\
& =\mathrm{T}_{1}^{\prime}\left(v_{1}\right) \cdots \mathrm{T}_{n}^{\prime}\left(v_{n}\right)=\mathrm{T}^{\prime}\left(v_{1}, \ldots, v_{n}\right)
\end{aligned}
$$

which is our claim.

Proposition 10. If $\mathrm{T}, \mathrm{T}^{\prime} \in E(G)$, then there exists an element $l \in \mathcal{E}_{n}$ such that, with respect to the coinduced action, $\mathrm{T}^{\prime}=\mathrm{T}^{l}$.

Proof. Take $l=m_{n-1} \cdots m_{1}$, where $m_{i} \in \mathcal{C}_{i}$ are given in Lemma 9 . 


\section{The iterated Frobenius EMBEDding}

Assume that

$$
1 \rightarrow K \hookrightarrow G \underset{\pi}{\rightarrow} Q \rightarrow 1
$$

is an exact sequence of groups, so that $Q \cong G / K$, and that $K$ is a subgroup of a group $R$. It is known that it is possible to embed $G$ in $R \imath Q$ [6]. Choose a restricted transversal $\mathrm{T}: Q \rightarrow G$ of $K$ in $G$, then the embedding is given by the formula

$$
\epsilon_{\mathrm{T}, R}(g)=\left(\pi(g), f_{g}\right)
$$

where the function $f_{g}: Q \rightarrow R$ is defined by $f_{g}(q)=\mathrm{T}\left(q \cdot \pi(g)^{-1}\right) \cdot g \cdot(\mathrm{T}(q))^{-1}$. If $\mathrm{T} \in E(G)$ we define the embedding $\theta_{\mathrm{T}, i}: G_{i} \rightarrow \mathcal{G}_{i}$ by recursion (see [7]). Put

$$
\theta_{\mathrm{T}, i}(g)= \begin{cases}\pi_{1}(g) \in V_{1}=\mathcal{G}_{1} & \text { if } i=1, \\ \epsilon_{\mathrm{T}_{i}, \mathcal{G}_{i-1}}(g) \in \mathcal{G}_{i-1} \prec V_{i}=\mathcal{G}_{i} & \text { if } i>1,\end{cases}
$$

where, in defining $\epsilon_{\mathrm{T}_{i}, \mathcal{G}_{i-1}}$, we think of $G_{i-1}$ embedded in $\mathcal{G}_{i-1}$ via $\theta_{\mathrm{T}, i-1}$. The homomorphism $\theta_{\mathrm{T}}=\theta_{\mathrm{T}, n}$ is called an iterated Frobenius embedding with respect to $\mathrm{T}$, and it is a regular embedding of $G$ in the permutation group $\mathcal{G}_{n}$ acting on $|G|$ points. In fact we have

Lemma 11. Assume that $\mathrm{T}: D_{1, n} \rightarrow G$ is a restricted exponential function, then for all $g \in G$ and for all $d \in D_{1, n}$, the equality $\mathrm{T}(d) g=\mathrm{T}\left(d^{\theta} \mathrm{T}(g)\right)$ holds.

Proof. If $n=1$, then $D_{1,1}=V_{1}$ and $\mathrm{T}(d) g=\mathrm{T}_{1}\left(d+\pi_{1}(g)\right)=\mathrm{T}\left(d^{\theta} \mathrm{T}(g)\right)$. If $n>1$ write $d=\left(\bar{d}, v_{n}\right)$, with $\bar{d} \in D_{1, n-1}$ and $v_{n} \in V_{n}$, and put $\overline{\mathrm{T}}(\bar{d})=\mathrm{T}(\bar{d}, 0)$. Then $\overline{\mathrm{T}}: D_{1, n-1} \rightarrow G_{n-1}$ is a restricted exponential function for $G_{n-1}$ such that $\mathrm{T}\left(\bar{d}, v_{n}\right)=\overline{\mathrm{T}}(\bar{d}) \mathrm{T}_{n}\left(v_{n}\right)$ and $\theta_{\mathrm{T}, n-1}=\theta_{\overline{\mathrm{T}}}$. Using induction on $n$ we can assume that the lemma holds for the exponential function $\overline{\mathrm{T}}$. Thus

$$
\begin{aligned}
\mathrm{T}(d) g & =\overline{\mathrm{T}}(\bar{d})\left(\mathrm{T}_{n}\left(v_{n}\right) g \mathrm{~T}_{n}\left(v_{n}+\pi_{n}(g)\right)^{-1}\right) \mathrm{T}_{n}\left(v_{n}+\pi_{n}(g)\right) \\
& =\overline{\mathrm{T}}\left(\bar{d}^{\theta} \bar{T}^{\left(\mathrm{T}_{n}\left(v_{n}\right) g \mathrm{~T}_{n}\left(v_{n}+\pi_{n}(g)\right)^{-1}\right)}\right) \mathrm{T}_{n}\left(v_{n}+\pi_{n}(g)\right) \\
& =\mathrm{T}\left(\bar{d}^{\theta} \bar{T}^{\left(\mathrm{T}_{n}\left(v_{n}\right) g \mathrm{~T}_{n}\left(v_{n}+\pi_{n}(g)\right)^{-1}\right)}, v_{n}+\pi_{n}(g)\right) \\
& =\mathrm{T}\left(\left(\bar{d}, v_{n}\right)^{\left(\pi_{n}(g), f\right)}\right)=\mathrm{T}\left(d^{\theta \mathrm{T}(g)}\right),
\end{aligned}
$$

where $f(i)=\theta_{\mathrm{T}, n-1}\left(\mathrm{~T}_{n}(i-\pi(g)) g \mathrm{~T}_{n}(i)^{-1}\right)$.

Corollary 12. Let $\mathrm{T}, \mathrm{T}^{\prime} \in E(G)$, then there exists an element $l \in \mathcal{E}_{n}$ such that $\left(\theta_{\mathrm{T}}(g)\right)^{l}=\theta_{\mathrm{T}^{\prime}}(g)$ for all $g \in G$. Hence $\theta_{\mathrm{T}}(G)$ and $\theta_{\mathrm{T}^{\prime}}(G)$ are conjugate subgroups of $\mathcal{G}_{n}$.

Proof. Let $l \in \mathcal{E}_{n}$ as in Proposition 10. For all $d \in D_{1, n}$ and $g \in G$ we have

$$
\mathrm{T}^{\prime}\left(d^{\theta \mathrm{T}^{\prime}}(g)\right)=\mathrm{T}^{\prime}(d) g=\mathrm{T}\left(d^{l^{-1}}\right) g=\mathrm{T}\left(d^{l^{-1} \theta_{\mathrm{T}}(g)}\right)=\mathrm{T}^{\prime}\left(d^{l^{-1} \theta_{\mathrm{T}}(g) l}\right) .
$$

Since $\mathrm{T}^{\prime}$ is injective and $\mathcal{G}_{n}$ is faithful on $D_{1, n}$, the thesis follows.

If $\mathrm{T} \in E(G)$ and $B\left(v_{j+1}, \ldots, v_{n}\right) \in F_{j, n}$, with $1 \leq j<n$, is a permutation block for $\mathcal{G}_{n}$ (see Proposition 5$)$, then $\mathrm{T}\left(B\left(v_{j+1}, \ldots, v_{n}\right)\right)=G_{j} \mathrm{~T}\left(0, \ldots, 0, v_{j+1}, \ldots, v_{n}\right)$ is a coset of $G_{j}$ in $G$. It is obvious that all such cosets can be described in this way. By Lemma 11 it follows that $\rho_{j, n}$ restricted to $\theta_{\mathrm{T}}(G)$ is the regular representation when $j=0$ and it is equivalent to the representation of $G$ acting on the cosets of $G_{j}$ in $G$, when $1 \leq j<n$. Since the subgroups $G_{j}$ are normal in $G$ we have, as an immediate consequence, the following proposition. 
Proposition 13. For all $g \in G$, the elements $\theta_{\mathrm{T}}(g)$ are diagonal in $\mathcal{G}_{n}$.

With the same argument we see that $\operatorname{wt}\left(\theta_{\mathrm{T}}(g)\right) \leq j$ if and only if $\theta_{\mathrm{T}}(g) \in$ $\operatorname{ker}\left(\rho_{j, n}\right)=\mathcal{B}_{j}$ i.e. $g \in G_{j}$ which is equivalent to $\operatorname{wt}(g) \leq j$. This can be stated as a proposition.

Proposition 14. wt $(g)=\operatorname{wt}\left(\theta_{\mathrm{T}}(g)\right)$ for all $g \in G$.

Assume that $G$ is a finite group and $H \leq G$ a subgroup, then the permutation character induced by $H$ to $G$ is denoted by $\mathbf{1}_{H}^{G}$. We shall use the well known formula

$$
\mathbf{1}_{H}^{G}(g)=\frac{\left|\mathrm{C}_{G}(g)\right| \cdot\left|g^{G} \cap H\right|}{|H|}
$$

where $g^{G}$ is the conjugacy class in $G$ of the element $g$ (see [4]). An immediate consequence is the following lemma.

Lemma 15. Assume that $G$ is a finite group and $H, K \leq G$ are subgroups. Then $\mathbf{1}_{H}^{G}=\mathbf{1}_{K}^{G}$ if and only if $\left|g^{G} \cap H\right|=\left|g^{G} \cap K\right|$ for all $g \in \bar{G}$.

We shall say, as in the introduction, that two subgroups $H$ and $K$ of a finite group $G$ are linked in $G$ if $\mathbf{1}_{H}^{G}=\mathbf{1}_{K}^{G}$.

Let $G$ be a finite group and $\Sigma=\left\{G_{i}\right\}_{i=0}^{n}$ an invariant series of $G$ with abelian factors $G_{i} / G_{i-1}$ of prime exponent $p_{i}$. Let also $p_{0}=1$. If $g \in G$ let $i_{1}=\operatorname{wt}(g)$, and recursively define $i_{k}=\operatorname{wt}\left(g^{p_{i_{1}} \ldots p_{i_{i-1}}}\right)$. The sequence

$$
\left\{i_{k}\right\}_{i=1}^{n}
$$

is called the weight sequence of the element $g$ with respect to $\Sigma$.

Theorem 16. Assume that $G$ and $H$ are two linked subgroups of a finite soluble group $L$. Then there exist invariant series $\left\{G_{i}\right\}_{i=1}^{n}$ of $G$ and $\left\{H_{i}\right\}_{i=1}^{n}$ of $H$ with abelian factors of prime exponent and a bijection $f: G \rightarrow H$, such that $g$ and $f(g)$ have the same weight sequence.

Proof. Let $\left\{L_{i}\right\}_{i=1}^{r}$ be a principal series of $L$ and let $\left\{G_{i}\right\}_{i=1}^{n}$ be the series obtained intersecting $\left\{L_{i}\right\}_{i=1}^{r}$ with $G$ and eliminating duplications. The series $\left\{G_{i}\right\}_{i=1}^{n}$ has abelian factors of prime exponent. For $i=1, \ldots, n$, let $M_{i}$ be the normal closure of $G_{i}$ in $L$. If $G_{i}=L_{s} \cap G$, then clearly $M_{i} \leq L_{s}$ and so $G_{i}=M_{i} \cap G$. Put $H_{i}=M_{i} \cap H$. We also have $H_{i}=L_{s} \cap H$. In fact $H_{i}=M_{i} \cap H \leq L_{s} \cap H$. To see the opposite inclusion let $h \in L_{s} \cap H$, then, by Lemma $15, h^{L}$ intersects $G$, so $\emptyset \neq h^{L} \cap G \subseteq L_{s} \cap G=G_{i}$ which implies $h \in M_{i} \cap H$. Again by Lemma 15 every conjugacy class of $L$ intersects $G_{i}$ and $H_{i}$ in the same number of elements. It follows that $\left|G_{i+1} / G_{i}\right|=\left|H_{i+1} / H_{i}\right|$. This implies that $\left\{H_{i}\right\}_{i=1}^{n}$ is obtained by intersecting $\left\{L_{i}\right\}_{i=1}^{r}$ with $H$ and so the factors $H_{i+1} / H_{i}$ are abelian. We have also that the exponent of $H_{i+1} \bmod M_{i}$ is equal to the exponent of $G_{i+1} \bmod M_{i}$, which is a prime integer. Hence $\left\{H_{i}\right\}_{i=1}^{n}$ is a series of normal subgroups of $H$ with abelian factors of prime exponent. Moreover, since $\left|g^{L} \cap G\right|=\left|g^{L} \cap H\right|$ for all $g \in G$, there exists a bijection $f: G \rightarrow H$ such that $f(g)$ is conjugate to $g$ in $L$. Then, for all $k \in \mathbb{Z}$, the element $f(g)^{k}$ is conjugate to $g^{k}$ in $L$. Hence $\operatorname{wt}\left(g^{k}\right)=\operatorname{wt}\left(f(g)^{k}\right)=\min \left\{i: g^{k} \in M_{i}\right\}$, so $f$ preserves the weight sequences.

Remark. With the notation as in the previous theorem it is easy to verify that $g$ has the same weight sequence as $f(g)$ if and only if for all $k \in \mathbb{Z}$ there holds $\operatorname{wt}\left(f\left(g^{k}\right)\right)=\operatorname{wt}\left(g^{k}\right)$. The sufficiency of the condition is trivial. Conversely assume 
that $g$ and $f(g)$ have the same weight sequence. Note that, if $\left(k, p_{i_{1}}\right)=1$, then $\operatorname{wt}\left(g^{k}\right)=\operatorname{wt}(g)=\operatorname{wt}(h)=\operatorname{wt}\left(h^{k}\right)$. Otherwise $g^{k}$ is a power of $g^{p_{i_{1}}}$. Since clearly $i_{2}=\operatorname{wt}\left(g^{p_{i_{1}}}\right)=\operatorname{wt}\left(f(g)^{p_{i_{1}}}\right)$ then the claim follows by induction on $\operatorname{wt}(g)$.

Let $V$ be a finite elementary abelian group of order $q=p^{r}$, for some prime $p$ and some positive integer $r . V$ can be identified with the additive group of the Galois field $\mathbb{F}(q)$. The semidirect product $A(q)=\mathbb{F}(q)^{*}[\mathbb{F}(q)]$ acts faithfully on $V$ as a one-dimensional affine group: $x^{(a, b)}=a x+b$. For $1 \leq j \leq n$ let $q_{j}=\left|V_{j}\right|$; we can then form the iterated wreath product of permutation groups:

$$
\mathcal{A}_{h, k}=\left(\cdots\left(A\left(q_{h}\right) \imath_{V_{h+1}} A\left(q_{h+1}\right) \imath_{V_{h+2}} \cdots\right) \imath_{V_{k}} A\left(q_{k}\right)\right) .
$$

We shall put, by definition, $\mathcal{A}_{n}=\mathcal{A}_{1, n}$.

The group $\mathcal{A}_{h, k}$ is a permutation group over $D_{h, k}$. Using Lemma 3 we find easily, for $h \leq i<k$, that $\mathcal{A}_{h, k}=\mathcal{A}_{h, i} 2_{D_{i+1, k}} \mathcal{A}_{i+1, k}$. We shall denote again by $\rho_{i, k}: \mathcal{A}_{h, k} \rightarrow \mathcal{A}_{i+1, k}$ the canonical projection, since it corresponds to the permutation representation of $\mathcal{A}_{h, k}$ on the block system $F_{i, k}$ obtained reasoning as in Proposition 5.

Lemma 17. Let $g$ and $g^{\prime}$ be two diagonal elements having the same weight sequence. Then $g$ and $g^{\prime}$ are conjugate elements of $\mathcal{A}_{n}$.

Proof. If $n=1$, then $A\left(q_{1}\right)$ acts transitively on $V_{1} \backslash\{0\}$ by conjugation. Two elements $g, g^{\prime} \in \mathcal{G}_{1}$ are then conjugate in $\mathcal{A}_{1}$ if and only if they are both trivial or not, hence if and only if $\operatorname{wt}\left(g^{r}\right)=\operatorname{wt}\left(g^{\prime r}\right)$ for all $r \in \mathbb{Z}$. We now assume that $n>1$ and argue by induction on $n$. We consider two cases:

(a) $\operatorname{wt}(g)=\operatorname{wt}\left(g^{\prime}\right)<n$,

(b) $\operatorname{wt}(g)=\operatorname{wt}\left(g^{\prime}\right)=n$.

In case (a) we show that $g$ and $g^{\prime}$ are conjugate in the base subgroup of $\mathcal{A}_{n}=$ $\mathcal{A}_{n-1} 2_{D_{n, n}} A\left(q_{n}\right)$. Let $\operatorname{wt}(g)=\operatorname{wt}\left(g^{\prime}\right)=h \leq n-1<n$, then $g$ and $g^{\prime}$ are in the kernel of the permutation representation $\rho_{n-1, n}$. Write $\mathcal{A}_{n}=\mathcal{A}_{n-1}{ }_{{ }^{2} D_{n, n}} A\left(q_{n}\right)$, then referring to such a decomposition, we can write $g=(1, a)$ and $g^{\prime}=(1, b)$ with $a, b: D_{n, n} \rightarrow \mathcal{G}_{t}$. By Proposition 7 , the elements $a(i)$ and $b(i)$ are diagonal and $\operatorname{wt}\left(a(i)^{k}\right)=\operatorname{wt}\left(b(i)^{k}\right)$, for all $k \in \mathbb{Z}$. By the remark following Theorem 16, they satisfy the inductive hypotheses and there exist elements $c(i) \in \mathcal{A}_{n-1}$ such that $a(i)^{c(i)}=b(i)$. This defines a function $c: D_{n, n} \rightarrow \mathcal{G}_{t}$. Put $h=(1, c)$, then $g^{h}=g^{\prime}$. The element $h$, belonging to $\operatorname{ker} \rho_{n-1, n}$, is contained in the base subgroup of $\mathcal{A}_{n}=\mathcal{A}_{n-1}$ 乙 ${ }_{-} D_{n, n} A\left(q_{n}\right)$.

In case (b) let $g=(u, a)$ and $g^{\prime}=(v, b)$, with $u, v \in V_{n}$ and $a, b: V_{n} \rightarrow \mathcal{G}_{n-1}$. Choose an element $l \in A\left(q_{n}\right)$ such that $u^{l}=v$ and put $s=(l, 1), g^{\prime \prime}=g^{s}=\left(v, a^{l}\right)$. The element $g^{\prime \prime}$, being conjugate to $g$, has the same weight sequence as $g$ and $g^{\prime}$. Also $\phi\left(g^{\prime \prime}\right)=\phi(g)$, where $\phi: \mathcal{G}_{n} \rightarrow V_{n}$ is the canonical projection. Consider the elements $g^{\prime p_{n}}$ and $g^{\prime p_{n}}$. They are diagonal, by Proposition 7, and conjugate in the base subgroup of $\mathcal{A}_{n-1} \prec A_{q_{n}}$ by case (a). Then there exists an element $t \in \mathcal{A}_{n-1}^{V_{n}}$ in the base subgroup such that $\left(g^{\prime \prime p_{n}}\right)^{t}=g^{\prime p_{n}}$. Since we have the inclusions $\mathcal{A}_{n-1}^{V_{n}} \leq \mathcal{A}_{n-1} \prec V_{n} \leq \mathcal{A}_{n-1} \imath_{V_{n}} A\left(q_{n}\right)=\mathcal{A}_{n}$ we now apply Proposition 8 to the group $\mathcal{A}_{n-1}\left\{V_{n}\right.$ and its elements $g^{\prime \prime t}$ and $g^{\prime}$. We deduce that there exists an $h \in \mathcal{A}_{n-1}\left\{V_{n}\right.$ such that $g^{\text {sth }}=g^{\prime \prime \prime}=g^{\prime}$, and, since sth $\in \mathcal{A}_{n}$, the claim is proved. 
Theorem 18. Let $\left\{G_{i}\right\}_{i=1}^{n}$ and $\left\{H_{i}\right\}_{i=1}^{n}$ be two invariant series with abelian factors of prime exponents respectively of the finite groups $G$ and $H$. Assume that there exists a bijection $f: G \rightarrow H$ such that, for all $g \in G$, the elements $g$ and $f(g)$ have the same weight sequence with respect to the above series. Let $\mathrm{T} \in E(G)$ and $\mathrm{T}^{\prime} \in E(H)$ be two restricted exponential functions. Then $\theta_{\mathrm{T}}(G)$ and $\theta_{\mathrm{T}^{\prime}}(H)$ are linked in $\mathcal{A}_{n}$.

Proof. By Propositions 7, 13 and 14, the elements $\theta_{\mathrm{T}}(g)$ and $\theta_{\mathrm{T}^{\prime}}(f(g))$ are conjugate in $\mathcal{A}_{n}$. Since $f$ is a bijection, we find that each $\mathcal{A}_{n}$-conjugacy class intersects both $\theta_{\mathrm{T}}(G)$ and $\theta_{\mathrm{T}^{\prime}}(H)$ in the same number of elements. The thesis then follows from Lemma 15.

We can summarize what we have proved in the following theorem.

Theorem 19. Let $G$ and $H$ be two soluble finite groups, then the following are equivalent:

(i) There exists a finite soluble group $L$ and embeddings $\sigma: G \rightarrow L$ and $\tau: H \rightarrow L$ such that $\mathbf{1}_{\sigma(G)}^{L}=\mathbf{1}_{\tau(H)}^{L}$.

(ii) There exist invariant series $\left\{G_{i}\right\}_{i=1}^{n}$ of $G$ and $\left\{H_{i}\right\}_{i=1}^{n}$ of $H$ with abelian factors of prime exponent, such that $\mathbf{1}_{\theta_{\mathrm{T}}(G)}^{\mathcal{A}_{n}}=\mathbf{1}_{\theta_{\mathrm{T}^{\prime}}(H)}^{\mathcal{A}_{n}}$ for all pairs of restricted exponential functions $\mathrm{T} \in E(G)$ and $\mathrm{T}^{\prime} \in E(H)$.

(iii) There exist invariant series $\left\{G_{i}\right\}_{i=1}^{n}$ of $G$ and $\left\{H_{i}\right\}_{i=1}^{n}$ of $H$ with abelian factors of prime exponent and a bijection $f: G \rightarrow H$ such that, for all $g \in G$, the elements $g$ and $f(g)$ have the same weight sequence with respect to the series above.

\section{A COUnTEREXAMPle AND SOME OPEN QUESTIONS}

From the results contained in [1] it is easy to see that, if $G$ and $H$ are two nilpotent groups inducing the same permutation character in some nilpotent finite overgroup, that there exist central series, say $\left\{G_{i}\right\}_{i=1}^{n}$ of $G$ and $\left\{H_{i}\right\}_{i=1}^{n}$ of $H$, such that $G_{i} / G_{i-1} \cong H_{i} / H_{i-1}=V_{i}$ is cyclic of order $p$ and $\mathbf{1}_{\theta_{\mathrm{T}}(G)}^{\mathcal{G}_{n}}=\mathbf{1}_{\theta_{\mathrm{T}^{\prime}}(H)}^{\mathcal{G}_{n}}$. It would be interesting to know if the group $\mathcal{G}_{n}$ works in place of $\mathcal{A}_{n}$ also in solvable cases. In fact $\left|\mathcal{G}_{n}\right|$ has the property of having the same prime divisors as $|G|$. The answer is no and we give a counterexample: there exist two solvable groups $H$ and $K$ which induce the same permutation character in a finite soluble group $L$ having the same prime divisors of $G$ and $H$, but for any choice of series $\left\{H_{i}\right\}_{i=0}^{n}$ of $H$ and $\left\{G_{i}\right\}_{i=0}^{n}$ of $G$ and respective restricted exponential functions $\mathrm{T}^{\prime}$ and $\mathrm{T}$, we have $\mathbf{1}_{\theta_{\mathrm{T}}(G)}^{\mathcal{G}_{n}} \neq \mathbf{1}_{\theta_{\mathrm{T}^{\prime}}(H)}^{\mathcal{G}_{n}}$.

Let $I$ be the $3 \times 3$ identity matrix and put $M=\left(\begin{array}{lll}0 & 0 & 1 \\ 1 & 0 & 0 \\ 0 & 1 & 0\end{array}\right)$ and $N=\left(\begin{array}{lll}2 & 0 & 0 \\ 0 & 1 & 0 \\ 0 & 0 & 1\end{array}\right)$, where the entries are in the Galois field $\mathbb{F}(7)$. Let $S=\langle a, b, c\rangle, T=\left\langle a^{\prime}, b^{\prime}, c^{\prime}\right\rangle$ and $Q=\left\langle a^{\prime \prime}, b^{\prime \prime}, c^{\prime \prime}\right\rangle$, where:

$$
\begin{aligned}
a & =\left(\begin{array}{lll}
0 & 0 & I \\
I & 0 & 0 \\
0 & I & 0
\end{array}\right), & a^{\prime}=a, & a^{\prime \prime}=a, \\
b & =\left(\begin{array}{ccc}
M & 0 & 0 \\
0 & 4 M & 0 \\
0 & 0 & 2 M
\end{array}\right), & b^{\prime}=\left(\begin{array}{ccc}
M & 0 & 0 \\
0 & M & 0 \\
0 & 0 & M
\end{array}\right), & b^{\prime \prime}=\left(\begin{array}{ccc}
M & 0 & 0 \\
0 & I & 0 \\
0 & 0 & I
\end{array}\right), \\
c & =\left(\begin{array}{ccc}
2 I & 0 & 0 \\
0 & 2 I & 0 \\
0 & 0 & 2 I
\end{array}\right), & c^{\prime}=c, & c^{\prime \prime}=\left(\begin{array}{ccc}
N & 0 & 0 \\
0 & I & 0 \\
0 & 0 & I
\end{array}\right) .
\end{aligned}
$$

The group $Q$ is isomorphic to $\left(C_{3} \succ C_{3}\right)$ ᄀ $C_{3}$ and is a Sylow 3 -subgroup of $\operatorname{GL}(9,7)$. The inclusions $S, T \leq Q$ are iterated Frobenius embeddings with respect to the 
central series $\Sigma: 1 \leq\langle c\rangle \leq\langle b, c\rangle \leq\langle a, b, c\rangle$ and $\Sigma^{\prime}: 1 \leq\left\langle c^{\prime}\right\rangle \leq\left\langle b^{\prime}, c^{\prime}\right\rangle \leq\left\langle a^{\prime}, b^{\prime}, c^{\prime}\right\rangle$. By a result contained in [1] the groups $S$ and $T$, having exponent $p$, induce the same permutation character to $Q$. Let $V=\mathbb{F}(7)^{9}$ be the natural module for $\operatorname{GL}(9,7)$, then let $G=S[V], H=T[V]$ and $L=Q[V]$. A direct counting argument shows:

$$
\left|(q, v)^{L} \cap G\right|=\left|q^{Q} \cap S\right| \frac{\left|C_{Q}(q) V\right|}{\left|C_{L}(q, v)\right|}=\left|q^{Q} \cap T\right| \frac{\left|C_{Q}(q) V\right|}{\left|C_{L}(q, v)\right|}=\left|(q, v)^{L} \cap H\right| .
$$

Hence $\mathbf{1}_{G}^{L}=\mathbf{1}_{H}^{L}$.

Let $\left\{G_{i}\right\}_{i=0}^{n}$ be an invariant series of $G$ with abelian factors of prime exponent and let $j$ be the smallest index such that $V \leq G_{j}$. We want to show that $V=G_{j}$. Assume, by contradiction, that 3 divides $\left|G_{j}\right|$. Let $W=V \cap G_{j-1}$ and $U$ be a 3-Sylow sequence of $G_{j}$. Without loss of generality we may assume $1 \neq U=S \cap G_{j}$. Since $V$ is a completely reducible $U$-module under conjugation, then $V=W \oplus X$, where $X \neq 0$ by the minimality of $j$. Moreover $G_{j} / G_{j-1}$ is of prime exponent and contains an isomorphic copy of $X$. We deduce that $U \leq G_{j-1}$. Let $g \in X$, then for all $s \in U$ we see $s^{g}=s[s, g] \in G_{j-1}$. Hence $[s, g] \in W \cap X=0$, and $X$ is a trivial $U$-module. By the definition of $S$ we see immediately that $V$ has no trivial $\mathrm{Z}(S)$-submodules, where $\mathrm{Z}(S)$ is the centre of $S$. So $\mathrm{Z}(S) \not Z U$, and this contradicts the fact that in an extraspecial 3 -group the centre is the unique minimal normal subgroup.

Let $N$ be a normal subgroup of $G$, then the series $1 \leq N \leq G$ can be refined to a chief series of $G$. By the argument in the last paragraph, we deduce that either $N \leq V$ or $V<N$. In particular $G$ has no normal subgroup of order 7 .

Lemma 20. If $g \in G_{1}$, then $\theta_{\mathrm{T}}(g) \in \mathcal{B}_{1}$. As a function from $D_{2, n}$ to $V_{1}, \theta_{\mathrm{T}}(g)$ maps $d$ to $\mathrm{g}^{\mathrm{T}(0, d)^{-1}}$. In particular, it maps $(0, \ldots, 0)$ to $\mathrm{g}$.

Proof. The claim is equivalent to

$$
\left(v_{1}, d\right)^{\theta_{\mathrm{T}}(g)}=\left(v_{1}+g^{\mathrm{T}(0, d)^{-1}}, d\right)
$$

for all $v_{1}$ in $V_{1}$. Since the exponential map is a bijection, the displayed equation is equivalent to

$$
\mathrm{T}\left(\left(v_{1}, d\right)^{\theta} \mathrm{T}(g)\right)=\mathrm{T}\left(v_{1}+g^{\mathrm{T}(0, d)^{-1}}, d\right) .
$$

By Lemma 11 the left hand side is $\mathrm{T}\left(v_{1}, d\right) g$, that is, $\mathrm{T}_{1}\left(v_{1}\right) \mathrm{T}(0, d) g$. The right hand side is $\mathrm{T}_{1}\left(v_{1}\right) \mathrm{T}_{1}\left(g^{\mathrm{T}(0, d)^{-1}}\right) \mathrm{T}(0, d)$. Since $\mathrm{T}_{1}$ is an identity map, this expression is equal to $\mathrm{T}_{1}\left(v_{1}\right) \mathrm{T}(0, d) g$, which proves the equality.

Assume, by contradiction, that there exist series $\left\{G_{i}\right\}_{i=0}^{n}$ of $G$ and $\left\{H_{i}\right\}_{i=0}^{n}$ of $H$ with restricted exponential functions $\mathrm{T}$ and $\mathrm{T}^{\prime}$ such that $\mathbf{1}_{\theta_{\mathrm{T}}(G)}^{\mathcal{G}_{n}}=\mathbf{1}_{\theta_{\mathrm{T}^{\prime}}(H)}^{\mathcal{G}_{n}}$. We have seen that there exists an index $j$ such that $G_{j}=V$, and by Lemma 15 , there is a bijection $f: G \rightarrow H$ such that $\theta_{\mathrm{T}^{\prime}}(f(g))$ is conjugate to $\theta_{\mathrm{T}}(g)$ in $\mathcal{G}_{n}$, then $\left|H_{j}\right|=\left|G_{j}\right|$, and hence $H_{j}=V$ too.

Since $H_{1} \leq V$ and $T$ is abelian with $\mathbb{F}(7)$ as splitting field for $T$, the minimal normal subgroups of $H$ contained in $H_{1}$ are all cyclic: let $\left\langle h_{1}\right\rangle$ be one of them. We know from Lemma 20 that $\theta_{\mathrm{T}^{\prime}}\left(h_{1}\right)$ is a function from $D_{2, n}$ to $V_{1}$ and all its values are $H$-conjugate to $h_{1}$. Since $\left\langle h_{1}\right\rangle$ is normal in $H$, all these values lie in $\left\langle h_{1}\right\rangle$ : that is, $\theta_{\mathrm{T}^{\prime}}\left(h_{1}\right) \in\left\langle h_{1}\right\rangle^{D_{2, n}}$. Suppose that $h \in H$ and $\theta_{\mathrm{T}^{\prime}}(h) \in\left\langle h_{1}\right\rangle^{D_{2, n}}$. By Proposition 14 (ii), $h \in H_{1}$. Hence, by the last clause in Lemma 20, $h$ is the value 
of $\theta_{\mathrm{T}^{\prime}}(h)$ at $(0, \ldots, 0)$, so that $h \in\left\langle h_{1}\right\rangle$. Thus the assumption $\theta_{\mathrm{T}^{\prime}}(h) \in\left\langle h_{1}\right\rangle^{D_{2, n}}$ implies that $h \in\left\langle h_{1}\right\rangle$ : we have proved that

$$
\theta_{\mathrm{T}^{\prime}}(H) \cap\left\langle h_{1}\right\rangle^{D_{2, n}}=\left\langle\theta_{\mathrm{T}^{\prime}}\left(h_{1}\right)\right\rangle .
$$

Since $\left\langle h_{1}\right\rangle^{D_{2, n}}$ is normal in $\mathcal{G}_{n}$ (being normalized by $\mathcal{G}_{2, n}$ and centralized by $\mathcal{B}_{1}$ ), and since its intersection with $\theta_{\mathrm{T}^{\prime}}(H)$ has order 7, it follows from Lemma 15 that $\theta_{\mathrm{T}}(G) \cap\left\langle h_{1}\right\rangle^{D_{2, n}}$ is a normal subgroup of order 7 . This contradicts the fact that $G$ has no normal subgroup of order 7 , and so completes the proof.

In [1], Section 4, there is an example of two $p$-groups $H$ and $K$, that induce the same premutation character in some common finite overgroup but which cannot be embedded as linked subgroups of any finite $p$-group. Referring to that example the series $1 \leq \Omega_{1}(H) \leq H$ and $1 \leq \Omega_{1}(K) \leq K$ are invariant with abelian factors of exponent $p$ (see [5], page 324, for the definition of $\Omega_{1}$ ). Note that any bijection $f: H \rightarrow K$ such that $f(1)=1$ and $f\left(\Omega_{1}(H)\right)=\Omega_{1}(K)$ preserves the weight sequences with respect to previous series. So $H$ and $K$ can be embedded as linked subgroups in a solvable finite group. So if $H$ and $K$ are linked $p$-subgroups of a solvable group they need not be linked subgroups of a finite $p$-group. This example leads to the open question:

Q1. If $G$ and $H$ are linked $p$-subgroups of a finite group, must there exist a finite solvable group with linked subgroups isomorphic to $G$ and $H$ ?

More generally it would be interesting to know if it is sufficient to count the elements of all possible given orders of two finite groups to state that they can be embedded as linked subgroups in a solvable group. Indeed we do not have an analogous example as in [1], Section 4, so we leave as open also the following:

Q2. If $G$ and $H$ are linked solvable subgroups of a finite group, does there exist a finite solvable group with linked subgroups isomorphic to $G$ and $H$ ?

\section{REFERENCES}

1. A. Caranti, N. Gavioli, S. Mattarei, Subgroups of finite p-groups inducing the same permutation character, Comm. Algebra 22 (1994), 877-895. MR 94k:20033

2. C. Curtis, I. Reiner, Representation theory of finite groups and associative algebras, Interscience, 1962. MR 26:2519

3. W. Feit, Some consequences of the classification of finite simple groups, Proceedings, Symposia in Pure Mathematics 37 (1980). MR 82c:20019

4. R. M. Guralnick, Subgroups inducing the same permutation representation I, J. Algebra $\mathbf{8 1}$ (1983), 312-319. MR 84j:20010

5. B. Huppert, Endliche Gruppen I, Springer-Verlag, Berlin, 1967. MR 37:302

6. L. Kaloujnine, M. Krasner, Produit complet des groupes de permutations et le problème d'extension des groupes III, Acta Sci. Math. Szeged 14 (1951), 69-82. MR 14:242d

7. F. Leinen, Chief series and right regular representation of finite p-groups, J. Austral. Math. Soc. 44 (1988), 225-232. MR 88m:20040

8. R. Perlis, On the equation $\zeta_{K}(s)=\zeta_{K^{\prime}}(s)$, J. Number Theory 9 (1977), 342-360. MR 56:5503

9. T. Sunada, Riemannian coverings and isospectral manifolds, Ann. Math. 121 (1985), 169-186. MR 86h:58141

Dipartimento di Matematica Pura ed Applicata, Università de L'Aquila, via Vetoio, I-67010 Coppito (AQ), ITALy

E-mail address: gavioli@univaq.it 